\title{
MAGNETIC SUSCEPTIBILITY AND CHARGE STATE OF Gd IMPURITY IN $\mathrm{Pb}_{1-x} \mathrm{Sn}_{x} \mathrm{Te}\langle\mathrm{Gd}\rangle$ CRYSTALS
}

\author{
D.M. ZayachuK, R.D. IvanchuK, V.I. Kempnyk and V.I. Mikityuk \\ Dept. of Physical Electronics, Chernivtsi State University \\ 2 Kotsyubynsky St., Chernivtsi 274012, Ukraine
}

\begin{abstract}
The effective magnetic momentum $p_{\text {eff }}$ of Gd-impurities in the $\mathrm{Pb}_{1-x} \mathrm{Sn}_{x} \mathrm{Te}\langle\mathrm{Gd}\rangle$ crystals as a function of crystal composition $x$ with $0 \leq x \leq 0.25$ is studied. It is shown that with the increase in tin content in the crystals from 0 to $0.15, p_{\text {eff }}^{2}(\mathrm{Gd})$ increases slowly from 37 to 43 . Then $p_{\text {eff }}^{2}(\mathrm{Gd})$ increases abruptly to 63 value when $x=0.16 \pm 0.01$. For subsequent increase $x \geq 0.17 p_{\text {eff }}^{2}(\mathrm{Gd})$ remains constant at 63 . Possible reasons of observed changes of $p_{\text {eff }}(\mathrm{Gd})$ and possible mechanisms of influence of the $\mathrm{Pb}_{1-x} \mathrm{Sn}_{x}$ Te crystal field on the effective magnetic momentum value of Gd-impurities in the crystals under study are analysed.
\end{abstract}

PACS numbers: $75.50 . P p$

\section{Introduction}

It is a well-known fact that gadolinium in Gd-containing compounds is usually present as $\mathrm{Gd}^{3+}$, donating $5 d^{1}$ and $6 s^{2}$ electrons to bonds [1]. However, its behaviour as a substitution impurity is not so obvious. Of specific interest would be the situation when the substitution ion in the foreign crystal matrix is present in a state distinct from $\mathrm{Me}^{3+}$.

IV-VI compounds seem to be the most suitable for investigating the above situation since the ground state of the intrinsic components in the metal sublattice is $\mathrm{Me}^{2+}$ [2]. The present paper investigates the behaviour of $\mathrm{Gd}$ as an impurity in IV-VI systems using as an example $\mathrm{Pb}_{1-x} \mathrm{Sn}_{x} \mathrm{Te}\langle\mathrm{Gd}\rangle$ crystals.

\section{Experimental}

Measurements were made on samples, prepared from Bridgman grown $\mathrm{PL}_{1-x} \mathrm{Sn}_{x} \mathrm{Te}\langle\mathrm{Gd}\rangle$ crystals with $0 \leq x \leq 0.25$, doped with $\mathrm{Gd}$ during growth. Sample composition was determined by the electron microprobe technique. Only samples with $\mathrm{Gd}$ content nonuniformity smaller than $5 \%$ were selected for further studies. 


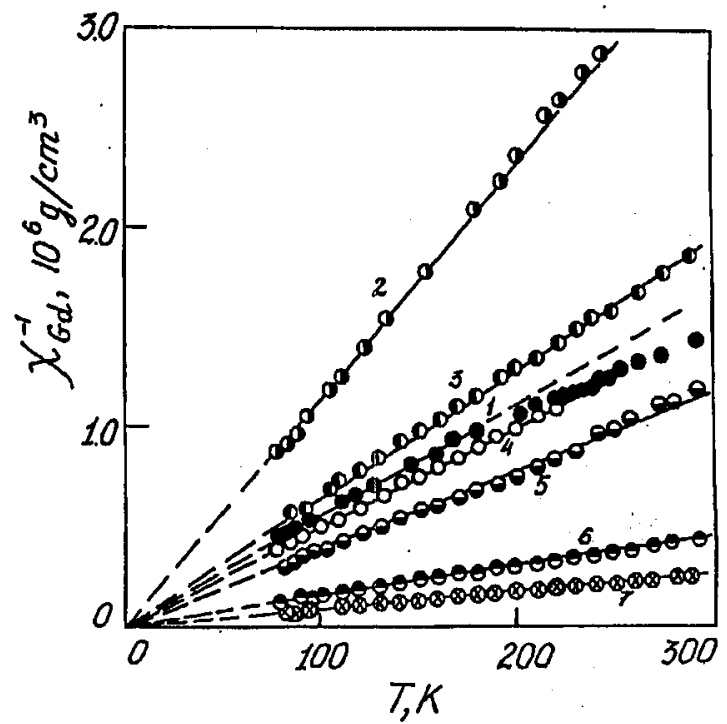

Fig. 1. Inverse magnetic susceptibility of Gd vs. temperature for $\mathrm{Pb}_{1-x} \mathrm{Sn}_{x} \mathrm{Te}\langle\mathrm{Gd}\rangle$ crystals. $x: 1-0,2-0.051,3-0.051,4-0.150,5-0.158,6-0.170,7-0.244$. $N(\mathrm{Gd}), 10^{20} \mathrm{~cm}^{-3}: 1-2.13,2-0.84,3-1.40,4-1.73,5-1.73,6-3.95$, $7-6.45$.

The total magnetic susceptibility $\chi$ of the crystals was measured using the Faraday method to within $1 \%$. The magnetic susceptibility of Gd $\chi_{\mathrm{Gd}}$ was determined by subtracting the $\chi$ value of undoped samples from the $\chi$ value of doped samples. Results for several $\mathrm{Pb}_{1-x} \mathrm{Sn}_{x} \mathrm{Te}\langle\mathrm{Gd}\rangle$ samples varying in composition are shown in Fig. 1.

The samples are observed to be paramagnetic within the temperature and composition ranges studied. The temperature variation of $\chi_{\mathrm{Gd}}$, especially for low temperatures, is well accounted for by the Curie law $\chi=C / T$ for $C=$ const. As some lead atoms are substituted by tin in the Gd crystalline environment, the temperature interval in which this law is valid extends into higher temperatures, as can be seen from Fig. 1.

Using the above results, we calculated the effective magnetic momentum of Gd impurity $p_{\text {eff }}(\mathrm{Gd})$ in $\mathrm{Pb}_{1-x} \mathrm{Sn}_{x} \mathrm{Te}\langle\mathrm{Gd}\rangle$ crystals using the magnetic susceptibility formula for paramagnetics [1]

$$
\chi=\frac{N \mu_{\mathrm{B}}^{2}}{3 k_{0} T} p_{\text {eff }}^{2},
$$

where $N$ is the concentration of paramagnetic atoms or ions, $\mu_{\mathrm{B}}$ is the Bohr magneton, and $k_{0}$ is the Boltzmann constant. The results are reported in Fig. 2.

As is seen from Fig. 2, $p_{\text {eff }}(\mathrm{Gd})$ is minimal at $x=0$ and grows slowly as $x$ increases from 0 to 0.15 . Then, in the narrow composition range $x=0.16 \pm 0.01$, a dramatic increase in $p_{\text {eff }}(\mathrm{Gd})$ is observed, and for $x \geq 0.17, p_{\text {eff }}(\mathrm{Gd})$ no longer depends on $x$ in the composition range studied. 


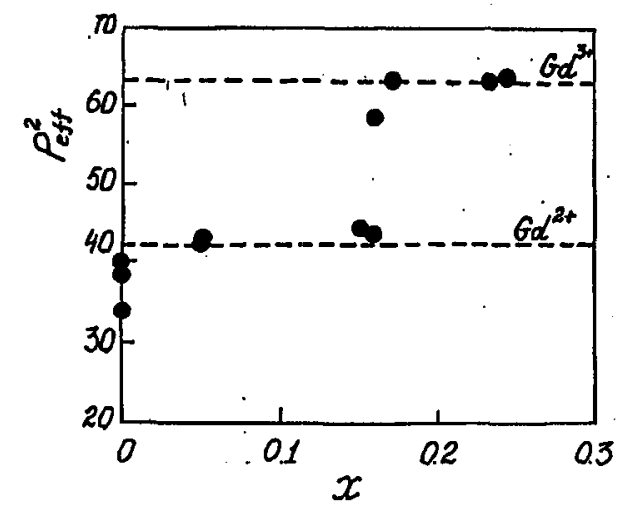

Fig. 2. The square of the effective magnetic momentum of Gd-impurities vs. $x$ for $\mathrm{Pb}_{1-x} \mathrm{Sn}_{x} \mathrm{Te}\langle\mathrm{Gd}\rangle$ crystals. Dotted lines correspond to $p_{\text {eff }}^{2}$ values of free ions of $\mathrm{Gd}^{2+}$ for infinite width multiplet and $\mathrm{Gd}^{3+}$.

\section{Discussion}

The effective magnetic momentum of many-electron ion is known to depend on its charge state. For chemical elements with wide multiplets, to which the rare earths belong, one can calculate as [1]

$$
\begin{aligned}
p_{\text {eff }}^{2}= & g_{J}^{2} J(J+1)+\frac{k_{0} T}{2(2 J+1)} \\
& \times\left\{\frac{\left[(S+L+1)^{2}-(J+1)^{2}\right]\left[(J+1)^{2}-(S-L)^{2}\right]}{(J+1) h \nu(J-1, J)}\right. \\
& \left.+\frac{\left[(S+L+1)^{2}-J^{2}\right]\left[J^{2}-(S-L)^{2}\right]}{J h \nu(J-1, J)}\right\},
\end{aligned}
$$

where $L, S, J$ are the orbital, spin and total momenta of a many-electron atom or ion, $g_{J}$ is the Lande factor, and $h \nu(J-1, J)$ is the width of the multiplet. For a free $\mathrm{Gd}^{3+}$ ion, $p_{\text {eft }}^{2}=63$, whereas for a free $\mathrm{Gd}^{2+}$ ion, $p_{\text {eff }}^{2}=42.67$ for an infinite width multiplet. For a finite width multiplet, the $p_{\text {eff }}^{2}(\mathrm{Gd})$ value would be somewhat higher.

It is easily seen that for $x \geq 0.17$, the $p_{\text {eff }}$ value of the Gd.ions in $\mathrm{Pb}_{1-x} \mathrm{Sn}_{x} \mathrm{Te}\langle\mathrm{Gd}\rangle$ is in excellent agreement with that of a free $\mathrm{Gd}^{3+}$ ion, which is completely dominated by the electrons of an unfilled $4 f$-shell. The $4 f$-shell is thus not affected by the crystalline environment of the Gd impurity in the materials studied, preserving its spin and orbital momenta.

The $p_{\text {eff }}^{2}$ values of the order of 37 to 43 . which are characteristic of $\mathrm{Gd}$ impurity in $\mathrm{Pb}_{1-x} \mathrm{Sn}_{x} \mathrm{Te}\langle\mathrm{Gd}\rangle$ for $0 \leq x \leq 0.15$, are close to that of a free $\mathrm{Gd}^{2+}$ ion. Hence, the Gd impurity in the above range of $x$ is in the charge state $\mathrm{Gd}^{2+}$, and in general, the crystalline environment of $\mathrm{Gd}$ affects its $p_{\text {eff }}$ value. It then follows that crystalline environment changes the $5 d$ electronic state of the $\mathrm{Gd}^{2+}$ ion, the amount of the change depending on the composition of a crystal matrix surrounding the impurity. 
The observed smooth variation of $p_{\text {eff }}(\mathrm{Gd})$ in $\mathrm{Pb}_{1-x} \mathrm{Sn}_{x} \mathrm{Te}\langle\mathrm{Gd}\rangle$ crystals with increasing $x$ and its dramatic increase at some critical $x_{c}$ value suggest that the reason of such changes is the presence of tin in the structure under study. The abrupt change of the Gd effective magnetic momentum is thought to be due to overlapping of external electronic shells of tin and the $\mathrm{Gd}^{2+}$ ion. This conclusion is based on the following simple estimates. The ionic radius of $\mathrm{Sn}^{2+}$ is $1.02 \AA$, the covalent radius is $1.4 \AA$ [3]. The radius of the $5 d$ electron shell of the Gd free ion is $5.33 \AA$ [1]. The sum of these radii would be of order $6.35-6.73 \AA$. This is comparable to the lattice constant of the solid solutions under study. The lattice constant is known to vary from 6.461 to $6.322 \AA$ as $x$ changes from 0 to 1 [4]. In $\mathrm{Pb}_{1-x} \mathrm{Sn}_{x}$ Te crystals, the lattice constant coincides with the second coordination sphere radius of a separate cation or anion sublattice (the materials under study have the $\mathrm{NaCl}$ crystal structure). Hence, if the tin content in a crystal is such that it inevitably gets into second coordination sphere of an arbitrary metal sublattice site which may be occupied by Gd impurity, them the external electronic shells of $\mathrm{Sn}$ and $\mathrm{Gd}^{2+}$ ions will come into contact for statistically uniform distribution of $\mathrm{Pb}_{1-x} \mathrm{Sn}_{x} \mathrm{Te}\langle\mathrm{Gd}\rangle$ crystal components. This will be the case when there is one Sn atom per six $\mathrm{Pb}$ atoms, which roughly corresponds to $x=0.15$ composition.

Therefore, in $\mathrm{Pb}_{1-x} \mathrm{Sn}_{x} \mathrm{Te}\langle\mathrm{Gd}\rangle$ crystals with statistically uniform composition, $\mathrm{Gd}$ will remain in the $\mathrm{Gd}^{2+}$ charge state as long as there is no direct contact between the external electronic shells of $\mathrm{Sn}$ and $\mathrm{Gd}$ ions. As $x$ increases and the above mentioned contact becomes inevitable, the $\mathrm{Gd}$ ion changes its state from $\mathrm{Gd}^{2+}$ to $\mathrm{Gd}^{3+}$. The conclusion is that in the materials studied the impurity state $\mathrm{Gd}^{2+}$ is more favourable than $\mathrm{Gd}^{3+}$. This appears to be due to the fact that in materials studied, $\mathrm{Pb}$ and $\mathrm{Sn}$ which are substituted by $\mathrm{Gd}$ in the metal sublattice are also present in the $\mathrm{Me}^{2+}$ state. The question why the effect of tin is to change the $\mathrm{Gd}^{2+}$ state into $\mathrm{Gd}^{3+}$ state calls for additional research.

\section{References}

[1] S.V. Vonsovskii, Magnetism, Nauka, Moskva 1971, p. 1032 (S.V. Vonsovskii, Magnetism, Wiley, New York 1974).

[2] G.A. Kalyugnaya, K.V. Kiseleva, in: Stoichiometry in Crystalline Compounds and its Effect on their Physical Properties, Trudi FIAN, Vol. 177, Nauka, Moskva 1987 (in Russian).

[3] C. Kittel, Introduction to Solid State Physics, Wiley, New York 1976.

[4] G. Nimtz, B. Schlicht, Narrow-gap Semiconductors, Springer, Berlin 1985, p. 1. 